\title{
Cycle Life Prediction for Rechargeable Battery based on Accelerated EODV Multi-phase Model: A Case on Nickel Hydrogen Battery
}

\author{
C.Y. Duan, Q.Z. HuangPeng, J.L. Zhou, G. Jin \\ College of Information System and Management, \\ National University of Defence Technology, Changsha, China.
}

Keywords: Life prediction, Multi-phased model, Accelerated equation

\begin{abstract}
In the health management of battery, cycle life prediction is an essential component. Before battery fail, the End-of-Discharge Voltage (EODV) reduce with the use. This paper proposed a multi-phase degradation model of EODV through failure mechanism analysis to evaluate the degree of degeneration of the battery at a specified future. The model includes parameter transformation and accelerated equation for different stress testing. By using such a model, the EODV of the cell over time for varied stress aging conditions can be accurately described. The model and inference methods are illustrated with a case application involving 15 nickel hydrogen cells in accelerated condition. The test results are as expected.
\end{abstract}

\section{Introduction}

Lifetime prediction of batteries is a key part in its health management. Aim at this, many methods and models such as capacity fading model [1] [2], end-of-discharge voltage (EODV) decreasing model were proposed. Decreasing of the EODV is a performance feature that indicates the state of battery is deterioration. We find the EODV of many types of battery shows a similar feature that is decline in multi-phase during charge-discharge cycle, e.g. nickel hydrogen $\left(\mathrm{N}-\mathrm{H}_{2}\right)$ batteries, lithium-ion batteries [3] and nickel cadmium batteries [4]. In this experiment, the EODV degradation curve of $\mathrm{N}-\mathrm{H}_{2}$ batteries can be separated into three phase, which corresponds to different aging mechanism. Degradation process like this can't be effectively traced by a single process, and an alternative model should be employed. This paper attempts to model EODV with a novel multi-phase procedure to make contribution in prediction of batteries' cycle life.

The $\mathrm{N}-\mathrm{H}_{2}$ battery cell is a rechargeable cell that has been widely used in space applications which require high reliability and long service life. These space applications include satellites operating for 15 or more years in geosynchronous orbits (GEO), as well as orbital spacecraft in low Earth orbits (LEO) which require many tens of thousands of charge and discharge cycles from batteries [5]. If nickel hydrogen battery serves as main power source in space vehicles, the residual life of these applications is greatly determined by the state of health of the $\mathrm{N}-\mathrm{H}_{2}$ battery. Hence, making accurate cycle life prediction of Nickel-hydrogen cell is extremely important in the reliability and safety estimation of the space applications.

\section{Experiment Design}

Fifteen nickel-hydrogen batteries applied in LEO satellite with a rated capacity of 40Ah has been tested in the experiment with a Maccor Series 4000 Automated Battery tester. Due to on-orbit working temperature is between $-15^{\circ} \mathrm{C}$ to $+25^{\circ} \mathrm{C}$, the cells were all placed in a closed temperatureregulated chamber and the temperature change of the surface of each cell and the chamber has been monitored.

Table 1.Test rules of the battery cycle life test

\begin{tabular}{|c|c|c|c|}
\hline Cells & DOD & Discharge in $0.6 C$ & Charge in $0.4 C$ \\
\hline $1,2,3,4,5$ & $40 \%$ & $40 \mathrm{~min}$ & $62.4 \mathrm{~min}$ \\
\hline $6,7,8,9,10$ & $60 \%$ & $60 \mathrm{~min}$ & $93.6 \mathrm{~min}$ \\
\hline $11,12,13,14,15$ & $80 \%$ & $80 \mathrm{~min}$ & $124.8 \mathrm{~min}$ \\
\hline
\end{tabular}


Each channel is connected with one $\mathrm{N}^{-\mathrm{H}_{2}}$ cell using the signal cable with 120 cores. The controlled accelerated factor involved in the experiment is DOD. These $15 \mathrm{~N}-\mathrm{H}_{2}$ batteries were placed in three different working condition levels that including 40\%DOD, 60\%DOD and $80 \%$ DOD. In other word, there were five batteries tested in each condition respectively. Every cell has been cycled continuously with the test rules shown in Table 1 for thirty-six weeks. The designed actual capacity of $\mathrm{Ni}-\mathrm{H}_{2}$ battery is $47 \mathrm{Ah}$, higher than the rated capacity. After the $\mathrm{N}-\mathrm{H}_{2}$ cells have been discharged with the desired DOD, the cells were recharged to the desired SOC with their commended C-rate.

\section{EODV Multi-phase model}

\section{EODV degradation model.}

Figure 1 shows the EODV curve in 60\% and 80\% DOD level obtained by designed experiment respectively. As can be seen, the curves present three phases change before cell's failure. Combining the experimental data with the aging mechanism of $\mathrm{Ni}^{-} \mathrm{H}_{2}$ battery, the EODV degradation curve could be divided into three phase: beginning fall, linear decreases and end-of-life drop. We believe that different aging mechanisms may dominate in a particular period leading to different cell aging behaviors in each stage.

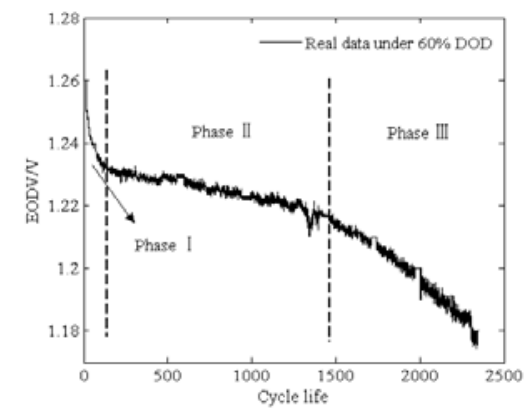

(a)

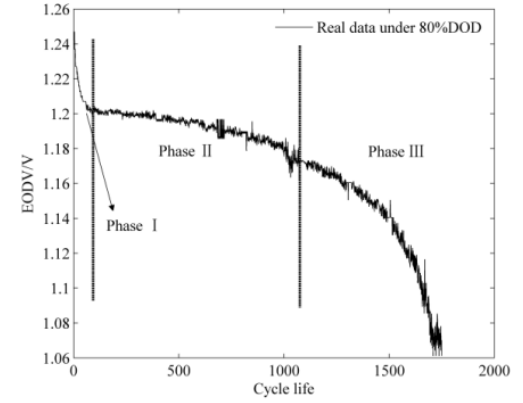

(b)

Figure 1.Real EODV curves in different accelerated test condition: (a) 60\% DOD condition, (b) 80\% DOD condition.

Beginning fall is the initial phase of $\mathrm{Ni}-\mathrm{H}_{2}$ batteries' life. When $\mathrm{Ni}-\mathrm{H}_{2}$ cell in original state, the active material is intensive in nickel substrate and the electrolyte is in good nature. However, affected by cell polarization effect, the EODV will show an exponential decline trend along with the charge-discharge cycles. But this process is not long, and finally achieves a more stable voltage. Equation (1) describes the EODV curve in this phase. Heret representsthe cycle, and $\beta_{1}$ and $\beta_{2}$ are unknown parameters, and $\beta_{3}$ represents the exponentially degradation rate of EODV.

$$
Y_{1}(t)=\beta_{1}+\beta_{2} \cdot \exp \left(\beta_{3} \cdot t\right), \quad t<t_{D 1}
$$

Linear decrease phase is a state which the $\mathrm{N}-\mathrm{H}_{2}$ battery maintain normal work, but actually their performance is deteriorated. The reason in this period the EODV shows a slow decline is mainly because the internal resistance of $\mathrm{Ni}-\mathrm{H}_{2}$ batteries is gradually increased Equation (2) represents this linear decrease phase. Here $t^{\prime}$ represents the cycle, $t^{\prime}=t-t_{D 1}$. And $\beta_{4}$ is a parameter on behalf of the linear degradation rate of the EODV. $\beta_{5}$ is an unknown parameter.

$$
Y_{2}\left(t^{\prime}\right)=\beta_{4} \cdot t^{\prime}+\beta_{5}, t_{D 1}<t^{\prime}<t_{D 2}
$$

End-of-life drop phase corresponds to the end of lifetime of $\mathrm{N}-\mathrm{H}_{2}$ battery, where its performance degraded exponentially. At this time, the nickel substrate surface bubbling while its surface become rough, loose and cracks increased. The electrochemical performance of $\mathrm{Ni}^{-} \mathrm{H}_{2}$ battery is also affected due to a large amount of active substances get into the aperture. The Nickel electrode becomes thicker and squeezes the diaphragm, result in the absorption of diaphragm decrease massively. EODV shows an exponential decline trend once again. Equation (3) could describe this terminal phase. Here $t^{\prime \prime}=t-t_{D 2}$. Here $\beta_{6}$ and $\beta_{8}$ are an unknown parameter. And $\beta_{7}$ represents the degradation rate of EODV. 


$$
Y_{3}\left(t^{\prime \prime}\right)=\beta_{6} \cdot \exp \left(\beta_{7} \cdot t^{\prime \prime}\right)+\beta_{8}, t^{\prime \prime}>t_{D 2}(3)
$$

On the basis of the above assumptions and inferences, the three-phase degradation model can be expressed as equation (4):

$$
Y(t)=Y_{1}(t) \cdot I_{\left[0, t_{D 1}\right)}(t)+Y_{2}\left(t-t_{D 1}\right) \cdot I_{\left[t_{D 1}, t_{D 2}\right)}(t)+Y_{3}\left(t-t_{D 2}\right) \cdot I_{\left[t_{D 2}, \infty\right)}(t)(4)
$$

Here, $I_{\left(t_{1}, t_{2}\right)}(t)$ is an indicative function as follow:

$$
I_{\left(t_{1}, t_{2}\right)}(t)=\left\{\begin{array}{l}
1, \text { when } t \in\left(t_{1}, t_{2}\right) \\
0, \text { when } t \notin\left(t_{1}, t_{2}\right)
\end{array}\right.
$$

\section{Model extended.}

Figure 2shows the EODV curve in 40\%DOD. The EODV curve doesn't show end-of-drop phenomenon at the end of test, which is different to the EODV curve in high stress. This is because the cycle life of $\mathrm{N}-\mathrm{H}_{2}$ battery in low DOD could up to the tens of thousands of times. So the $40 \%$ DOD batteries couldn't enter the end of life this time, result in exponential decline not appears to its EODV curve. We could get the two-phase model of the EODV as follow:

$$
Y(t)=Y_{1}(t) \cdot I_{\left[0, t_{D 1}\right)}(t)+Y_{2}\left(t-t_{D 1}\right) \cdot I_{\left[t_{D 1}, t_{D 2}\right)}(t)(6)
$$

However, the loss of the last phase makes the degradation model poor in extrapolation and not accurate in prediction of cycle life. We need to use parameter transform and accelerated equation to extend the EODV model in 40\%DOD.This method could also be used in other stress.

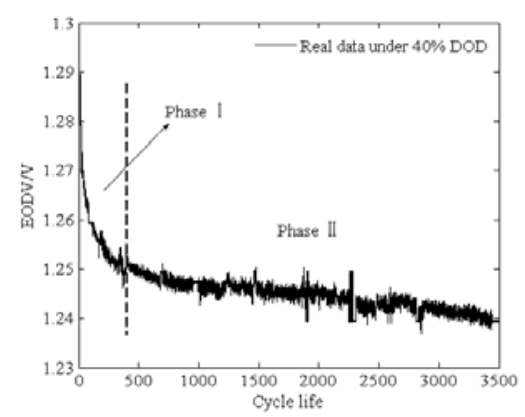

Figure 2. Real EODV curve in $40 \%$ DOD test condition

When degradation test data are gathered in accelerated or high stress conditions (such as elevated DOD), the relation between the level of stress and the model parameters could be established so that the parameters can be extrapolated to the lower stress conditions encountered in actual use of the item. Parameters $\left(\beta_{1 k}, \beta_{2 k}, \beta_{3 k}, \beta_{4 k}, \beta_{5 k}\right)^{T}(k=1,2,3)$ could be obtained through model fitting, but $\left(\beta_{6 k}, \beta_{7 k}, \beta_{8 k}\right)^{T}$ need be extrapolate by $\left(\beta_{6 k}, \beta_{7 k}, \beta_{8 k}\right)^{T}(k=2,3)$. Let the stress be described by a single quantitative measure which denote by $S$. We assume that the accelerated relations between the $S$ and the transformed parameters are linear as follows.

$$
F\left(\beta_{i}\right)=m_{i}+n_{i} \cdot S(7)
$$

Here $F\left(\beta_{i}\right)$ denotes the parameter transformations:

$$
\left\{\begin{array}{l}
F\left(\beta_{i}\right)=\beta_{i}, i=1,2,4,5,6,8 \\
F\left(\beta_{i}\right)=\ln \left|\beta_{i}\right|, i=3,7
\end{array}\right.
$$

The $m$ and $n$ are parameters in the accelerated function. It was difficult to judge whether these transformations were reasonable from a scientific point of view. Thus, the final judgment was to be based on the adequacy of the statistical fit. After parameters estimation and model extended, we finally get the degradation model of the EODV in different DOD stress. Supposed the failure threshold of the EODV as $T$, the pseudo-life $L_{i k}$ of the battery is:

$$
L_{i k}=\left\{t_{i} \mid Y_{i j k}\left(t_{i j k} \mid \widehat{\boldsymbol{\beta}}_{i k}\right)=T, t_{i} \geq 0\right\}
$$




\section{Simulation}

At begin, we modeling the EODV curve in 60\% and 80\%DOD using the three-phase model. The NONLIN procedure of the statistical software MATLAB R2011b was used to obtain the estimates $\widehat{\boldsymbol{\beta}}_{\boldsymbol{i} \boldsymbol{k}}$ for the ith item $(i=1, \ldots, 5)$ at the $k$ th depth of discharge level $(k=2,3)$ by minimizing the sum of the absolute value of squared deviations for each of the 10 items. According to Figure $1(\mathrm{a}), t_{D 12}$ is set to equal $1.23 \mathrm{~V}$ and $t_{D 22}$ is set to equal $1.20 \mathrm{~V}$, which means that if $(t)<t_{D 12}$, the battery is degrading in the first phase.If $t_{D 12}<Y(t)<t_{D 22}$, the battery is degrading in the second phase, or else in the third phase.And $t_{D 13}$ is set to equal $1.22 \mathrm{~V}$ as well as $t_{D 23}$ is set to equal $1.18 \mathrm{~V}$. Apply two-phase models to fit the EODV curve in 40\% DOD. At this time, $t_{D 11}$ sets to $1.25 \mathrm{~V}$, and $t_{D 21}$ sets to $1.22 \mathrm{~V}$. The $\mathrm{R}^{2}$ index which could measures the goodness of fit of a model was used to evaluate the quality of the results. Table 2 shows the $\mathrm{R}^{2}$ index for each cell items. The results show that the parameter estimates are reasonably consistent across items at each DOD level.

Table 2. $\mathrm{R}^{2}$ index for each item sin different DOD.

\begin{tabular}{|c|c|c|c|}
\hline & $\begin{array}{c}40 \% \\
\text { DOD }\end{array}$ & $60 \%$ DOD & $80 \%$ DOD \\
\hline 1 & 0.9882 & 0.9782 & 0.9914 \\
\hline 2 & 0.9856 & 0.9753 & 0.9897 \\
\hline 3 & 0.9875 & 0.9801 & 0.9892 \\
\hline 4 & 0.9831 & 0.9799 & 0.9892 \\
\hline 5 & 0.9844 & 0.9821 & 0.9906 \\
\hline
\end{tabular}

Table 3. Parameter estimation based on parameter transform and accelerated equation.

\begin{tabular}{|c|c|c|c|c|c|c|c|c|}
\hline & \multicolumn{7}{|c|}{ Parameter Estimation } \\
\hline DOD & $\beta_{2}$ & $\beta_{z}$ & $\beta_{x}$ & $\beta_{*}$ & $\beta_{s}$ & $\beta_{x}$ & $\beta_{z}$ & $\beta_{x}$ \\
\hline $40 \%$ & 1.2538 & 0.033992 & -0.01433 & $-2.80 \mathrm{E}-06$ & 1.251 & -0.014108 & $2.93 \mathrm{E}-04$ & 1.2998 \\
\hline $60 \%$ & 1.2281 & 0.043238 & -0.02928 & $-1.21 \mathrm{E}-05$ & 1.229 & -0.007422 & $9.34 \mathrm{E}-04$ & 1.2442 \\
\hline $80 \%$ & 1.2025 & 0.052484 & -0.05982 & $-2.14 \mathrm{E}-05$ & 1.207 & $-7.36 \mathrm{E}-04$ & 0.002974 & 1.1886 \\
\hline
\end{tabular}

In order to extend the two-phase model, we need to extrapolate its parameters. $\left(\beta_{1 i k}, \beta_{2 i k}, \beta_{3 i k}, \beta_{4 i k}, \beta_{5 i k}\right)^{T} \quad(k=1,2,3)$ could be calculated in three DOD conditions, $\operatorname{but}\left(\beta_{6 i 1}, \beta_{7 i 1}, \beta_{8 i 1}\right)^{T}$ need to be extrapolated. The plots for the transformed parameters appear in Figure 3.Each set of parameter estimates was calculated along with the depth of discharge. Based on the follow eight plots, the parameter transformations were tentatively identified. The plots in Figure 3 show roughly linear relationships between DOD and parameter transformations. The parameters of accelerated equation $F\left(\beta_{i}\right)=m_{i}+n_{i} \cdot S$ could be obtained by minimizing the total sum of squares for each of the above sets of values. Now, a quantitative relationship between DOD and parameters of EODV model is available. for all tested items in designed stresses. Note that the parameters of EODV degradation model in other stress level could be inferred in theoretically. 

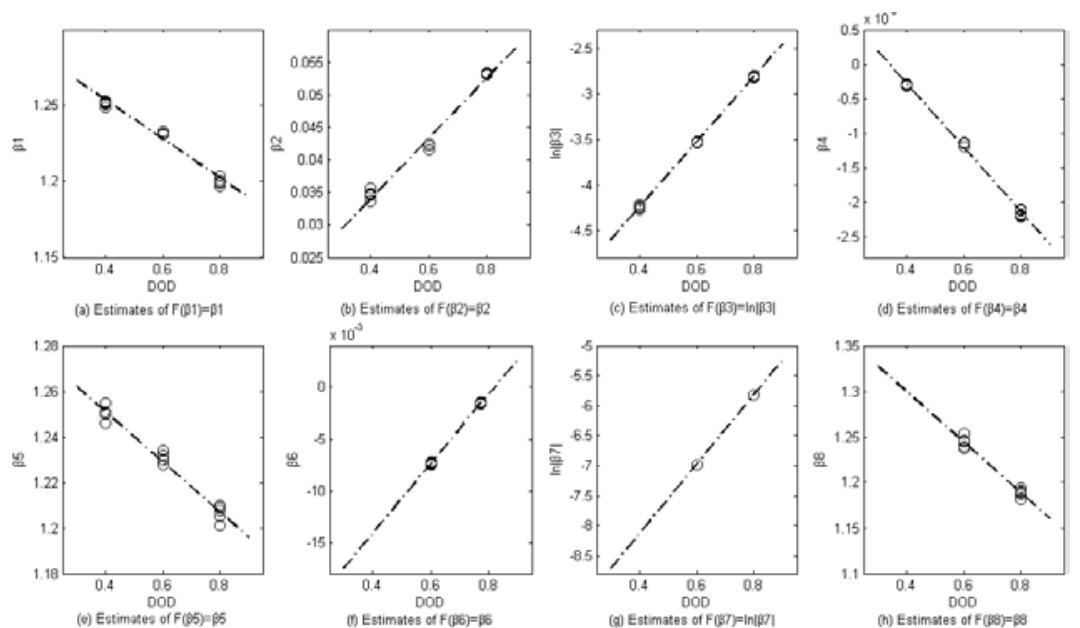

Figure 3.Plots of the transformed parameter estimates against the DOD fortest item.

Table 4. Cycle life prediction for the tested cells in different aging condition.

\begin{tabular}{|c|c|c|c|}
\hline & \multicolumn{3}{|c|}{ Test Condition } \\
\hline & $\begin{array}{c}40 \% \\
\text { DOD }\end{array}$ & $\begin{array}{r}60 \% \\
\text { DOD }\end{array}$ & $\begin{array}{c}80 \% \\
\text { DOD }\end{array}$ \\
\hline $\begin{array}{c}\text { Cycle } \\
\text { Life }\end{array}$ & 10432 & 3741 & 1866 \\
\hline
\end{tabular}

In the LEO application, the end of life was defined as $1.0 \mathrm{~V}$ of the EODV, in other words, if the EODV range value exceeds the threshold, the nickel hydrogen battery is thought to fail. So, this EODV degradation model can be used as the basis to predict the cell lifetime according to equation (10). The estimate of cell cycle life in different aging condition is presented in Table .

\section{Conclusion}

In this paper, we present a model to evaluate the cycle life of $\mathrm{N}-\mathrm{H}_{2}$ batteries whose EODV degradation process is obviously separated into three phases. To do this, we first model the batteries' EODV degradation path by multi-phase process in varied DOD level. Then, we estimate parameters of the model, and achieve the extrapolation of parameters through parameter transformation and accelerated equation. Finally, we applied this model to $15 \mathrm{~N}-\mathrm{H}_{2}$ cells, which demonstrates its efficiency.

We believe that the major contributions of this work are: (i) the model could generalized to other types of batteries, whose degradation path is similarly separated into different phases; (ii) the model in low stress level can be extended using the test data acquired from high stress level; and (iii) an approach to apply this model to real-world data and evaluate its effectiveness. We believe that the results presented in Section 4 clearly illustrate the importance of accurately modeling the degradation process. Future research directions include extending the model into more kind of batteries to validate it, and considering different stochastic process at different phase.

\section{Acknowledgments}

The author would like to express his sincere thanks to the National Science Foundation of China under grant no. 71271212 and no. 61273041 which supports this work.

\section{References}

[1] Linden. D., Reddy, T.B., Handbook of Batteries, McGraw-Hill Professional (2010).

[2] Long Lam, Pavol Bauer, Practical Capacity Fading Model for Li-Ion Battery Cells in Electric 
Vehicles, IEEE Transaction on Power Electronics, 28, 2013.

[3] Gang Ning, Branko N. Popov, Cycle Life Modeling of Lithium-Ion Batteries, Journal of The Electrochemical Society, 151(10) ( 2004)

[4] J.L. Sullivan, L. Gaines, Status of life cycle inventories for batteries,Energy Conversion and Management, 134-148, 58 (2012) .

[5] L.H. Thaller, A. Zimmerman, Nickel-Hydrogen Life Cycle Testing - Review and Analysis, American Institute of Aeronautics and AstronauticsAerospace Press (2003).

[6] H.S. Lim, S.A. Verzwyvelt, Long Life NickelElectrodesforA Nickel -Hydrogen Cell: Cycle Life Tests, Proceedings of the Eighteenth Intersociety Energy Conversion Engineering Conference, (1983).

[7] A. Zimmerman, Nickel-HydrogenBatteries- Principles and Practice, American Institute of Aeronautics and AstronauticsAerospace Press (2009). 